\title{
Some Periodic Continued Fractions With Long Periods
}

\author{
By C. D. Patterson and H. C. Williams*
}

\begin{abstract}
Let $p(D)$ be the period length of the continued fraction for $\sqrt{D}$. Under the extended Riemann Hypothesis for $2(\sqrt{D})$ one would expect that $p(D)=O\left(D^{1 / 2} \log \log D\right)$. In order to test this it is necessary to find values of $D$ for which $p(D)$ is large. This, in turn, requires that we be able to find solutions to large sets of simultaneous linear congruences. The University of Manitoba Sieve Unit (UMSU), a machine similar to D. H. Lehmer's DLS-127, was used to find such values of $D$. For example, if $D=46257585588439$, then $p(D)=$ 25679652. Some results are also obtained for the Voronoi continued fraction for $\sqrt[3]{D}$.
\end{abstract}

1. Introduction. Let $D$ be any positive integer. In Williams [7] it was pointed out that if $D$ is square-free, then $p(D)$, the period length of the continued fraction expansion of $\sqrt{D}$, should be bounded above by an expression of the form $c D^{1 / 2} \log \log D$. In fact, if

$$
f(D)= \begin{cases}D^{1 / 2} \log \log D & \text { for } D \equiv 1(\bmod 8) \\ D^{1 / 2} \log \log 4 D & \text { otherwise }\end{cases}
$$

we should have

$$
G(D)=p(D) / f(D)<k+o(1)
$$

under the extended Riemann Hypothesis for $\zeta_{\mathscr{X}}$ when $\mathscr{K}=\mathscr{2}(\sqrt{D})$. Here $k=3.7012$, but we expect by Lévy's Law that the smaller value $12 e^{\gamma} \log 2 / \pi^{2} \approx 1.50103$ could be used for $k$. In [7] values of $D\left(<2 \times 10^{9}\right)$ were examined in order to find large values of $G(D)$. The largest value found was that of $G(D)=1.040452$ for $D=$ 1492180699. In this paper we describe a further attempt to find values of $D$ for which $G(D)$ is large. We also describe some analogous work done in the case of Voronoi's algorithm in $2(\sqrt[3]{D})$.

2. Numerical Results. A glance at the results and tables given in [7] reveals that, in order to find values of $D$ for which $G(D)$ is likely to be large, one should examine integers of the form $q$ or $2 q$, where $q$ is a prime and $q \equiv-1(\bmod 4)$. Further, if $r_{i}$ is the $i$ th odd prime, one should also attempt to have the Legendre symbols

$$
\left(D / r_{i}\right)=1 \quad(i=1,2,3, \ldots, n)
$$

for as large a value of $n$ as possible. Thus, for each such $r_{i}$ we would want $D$ to belong to one of the $\left(r_{i}-1\right) / 2$ congruence classes such that $\left(D / r_{i}\right)=1$. To find such values of $D$ requires that we find solutions of large numbers of simultaneous

Received November 23, 1984.

1980 Mathematics Subject Classification. Primary 10-04, 10A32, 12A30.

* Research supported by NSERC of Canada Grant \# A7649 and the I. W. Killam Foundation. 
linear congruences, a problem best solved by using a number sieve (see Lehmer [3]). In Patterson and Williams [5] a very fast version of such a device, called the University of Manitoba Sieve Unit (UMSU), is described. This machine will solve such systems of congruences at the rate of $1.33 \times 10^{8}$ trials at a solution per second.

We searched for values of $D$ of four different types:

$\begin{array}{rll}\text { (i) } & D \equiv 3(\bmod 8) & D \text { prime } \\ \text { (ii) } & D \equiv 7(\bmod 8) & D \text { prime } \\ \text { (iii) } & D \equiv 6(\bmod 8) & D / 2 \text { prime } \\ \text { (iv) } & D \equiv 1(\bmod 8) & D \text { prime }\end{array}$

We examined values of $D$ of type (iv) to determine whether values of $G(D)$ would, as predicted by Shanks, tend to catch up to the larger values obtained for the other types of $D$. For each value of $n(1,2,3, \ldots)$ UMSU was programmed to search for the first $m$ (at least) values of $D$ of a given type. For $n \leqslant 32\left(r_{32}=137\right)$, we used 50 as our value of $m$. Because of the amount of time needed to go farther, we cut this value down to 10 for $33 \leqslant n \leqslant 36\left(r_{36}=157\right)$. In addition, for $n=37$ we used $m=7$ and $m=8$ for $D$ of type (i) and type (iii), respectively. For $D$ of type (iv) we used $m=10$ for $n=37$ and $m=4$ with $n=38$ for $D$ of type (ii).

After these numbers had been found, a job requiring many weeks of continuous use of UMSU, we computed the corresponding continued fraction period lengths $p(D)$ and the values of $G(D)$. We summarize our results in the four tables given below. We give only those numbers $D>2 \times 10^{9}$. Also, we print $D$ and its corresponding $p$ - and $G$-values only when $G(D)$ exceeds the value of $G(d)$ for all of our computed values of $d$ of the same type with $d<D$.

In Table 5 we present the values of $D$, from among those found by UMSU, with the largest $p(D)$ values. We give five such numbers for each $D$-type.

On examining these tables, we see that the values of $G(D)$ are certainly growing sufficiently slowly for (1.1) to hold. Further, the values of $G(D)$ for $D$ of type (iv) seem to be slowly catching up to those values for the other $D$-types.

TABLE $1 . D$ - Type (i)

\begin{tabular}{|r|r|r|}
\hline \multicolumn{1}{|c|}{$D$} & \multicolumn{1}{|c|}{$\mathrm{p}(\mathrm{D})$} & $G(D)$ \\
\hline 2186009851 & 151838 & 1.037297 \\
2287905811 & 155710 & 1.039131 \\
7528121899 & 288198 & 1.043420 \\
30738225571 & 603178 & 1.061828 \\
614886781051 & 2794390 & 1.063448 \\
1260977393659 & 4081590 & 1.076694 \\
55400066448211 & 28076486 & 1.078532 \\
\hline
\end{tabular}


In Table 6, we extend part of Table I of Lehmer, Lehmer and Shanks [4]. That is, for various values of $n$ we give the least prime $D \equiv 1(\bmod 8)$ such that $(2.1)$ holds. We also mention here that D. H. Lehmer had already found previously (but not published) the first six lines of this table.

TABLE 2. $D$ - Type (ii)

\begin{tabular}{|c|c|c|}
\hline$D$ & $P(D)$ & $G(D)$ \\
\hline 2763423391 & 170804 & 1.034456 \\
5097972751 & 234768 & 1.038196 \\
12095524039 & 366384 & 1.040132 \\
19672399231 & 471320 & 1.042810 \\
24880707679 & 536964 & 1.053362 \\
50151351559 & 772360 & 1.058250 \\
62324011759 & 864408 & 1.059728 \\
492210358039 & 2519212 & 1.074069 \\
4944598510471 & 8181752 & 1.075383 \\
22542868742839 & 17739532 & 1.076772 \\
46257585588439 & 25679652 & 1.081244 \\
\hline
\end{tabular}

TABLE 3. D - Type (iii)

\begin{tabular}{|r|r|r|}
\hline$D$ & $p(D)$ & $G(D)$ \\
\hline 2340752254 & 157036 & 1.035754 \\
7636279366 & 288766 & 1.037853 \\
8813799094 & 312690 & 1.044133 \\
8932573654 & 316434 & 1.049406 \\
31416841054 & 611088 & 1.063790 \\
6730689687166 & 9585044 & 1.076654 \\
13518648471574 & 13732410 & 1.081381 \\
\hline
\end{tabular}


TABLE 4. $D$ - Type (iv)

\begin{tabular}{|c|c|c|}
\hline D & $p(D)$ & $G(D)$ \\
\hline 18901431649 & 433383 & .996329 \\
22945498489 & 479525 & .997981 \\
23258723401 & 483919 & 1.000142 \\
28467424441 & 540685 & 1.007395 \\
37312059409 & 625233 & 1.013966 \\
40094470441 & 653345 & 1.021500 \\
163965430561 & 1348681 & 1.024427 \\
192052219969 & 1473213 & 1.032023 \\
2570329924369 & 5552441 & 1.033038 \\
2871842842801 & 5924695 & 1.041624 \\
8103297298321 & 10135403 & 1.049695 \\
457165855430761 & 79417945 & 1.055452 \\
\hline
\end{tabular}

3. Some Analogous Results for $\sqrt[3]{D}$. It is well-known that the regular continued fraction expansion of $\sqrt[3]{D}$ is never periodic; however, Voronoi's [6] continued fraction is periodic for cubic irrationalities. Let $\mathscr{K}=2(\sqrt[3]{D})$ be the pure cubic field formed by adjoining $\sqrt[3]{D}$ to the rationals $\mathscr{2}$, and let $\Delta$ be the discriminant of $\mathscr{K}$. Then, if $D$ is cube-free and $D=a b^{2}$ with $(a, b)=1$, we have

$$
\Delta= \begin{cases}-3 a^{2} b^{2} & \text { when } a^{2} \equiv b^{2}(\bmod 9) \\ -27 a^{2} b^{2} & \text { otherwise }\end{cases}
$$

If $\varepsilon_{0}$ is the fundamental unit of $\mathscr{K}, R\left(=\log \varepsilon_{0}\right)$ the regulator of $\mathscr{K}$, and $P$ the period of Voronoi's continued fraction, then by (8.3) of Williams [8], we get

$$
R>[P / 4] \log 2
$$

Unfortunately, we do not yet have a rule like Lévy's for this case, but it seems from empirical evidence that

$$
R \approx v P,
$$




\section{TABLE 5}

\begin{tabular}{|c|c|c|c|}
\hline Type & D & $p(D)$ & $G(D)$ \\
\hline \multirow{5}{*}{ (i) } & 152290419440611 & 46274886 & 1.062983 \\
\hline & 165427035605659 & 48190146 & 1.061386 \\
\hline & 206546921647291 & 54350198 & 1.069334 \\
\hline & 215226414830491 & 54450146 & 1.049121 \\
\hline & 300272328240091 & 65344634 & 1.063030 \\
\hline \multirow{5}{*}{ (ii) } & 133051755648751 & 42848636 & 1.054226 \\
\hline & 142368153139039 & 44889152 & 1.067078 \\
\hline & 146936775525439 & 45349180 & 1.060843 \\
\hline & 166290530163319 & 48736480 & 1.070583 \\
\hline & 174346066249111 & 49611996 & 1.063923 \\
\hline \multirow{5}{*}{ (iii) } & 246406633037854 & 57923528 & 1.041889 \\
\hline & 256397742215806 & 60536004 & 1.067108 \\
\hline & 285278695393246 & 64119584 & 1.070606 \\
\hline & 301938138430366 & 64551980 & 1.047187 \\
\hline & 350240722763374 & 70400728 & 1.059121 \\
\hline \multirow{5}{*}{ (iv) } & 229297977151681 & 54793321 & 1.034296 \\
\hline & 259853252349289 & 58673599 & 1.039268 \\
\hline & 273323976657169 & 60545353 & 1.045206 \\
\hline & 366525636221761 & 69241975 & 1.029650 \\
\hline & 457165855430761 & 79417945 & 1.055462 \\
\hline
\end{tabular}

where $1.12<v<1.13$. Thus, if we can bound $R$, we can certainly get a result like (1.1).

If $h$ is the class number of $\mathscr{K}$, we have

$$
h R=\frac{\sqrt{|\Delta|}}{2 \pi} \Phi(1)
$$

where

$$
\Phi(1)=\lim _{s \rightarrow 1} \zeta_{\mathscr{X}}(s) / \zeta(s)=\prod_{q} f(q) .
$$


TABLE 6

\begin{tabular}{|c|r|}
\hline $\mathrm{r}_{\mathrm{n}}$ & \multicolumn{1}{|c|}{ Least $\mathrm{D}$} \\
\hline 83 & 8114538721 \\
89 & 9176747449 \\
$97,101,103$ & 23616331489 \\
$107,109,113,127$ & 196265095009 \\
$131,137,139$ & 2871842842801 \\
149 & 26437680473689 \\
151 & 89436364375801 \\
$157,163,167$ & 112434732901969 \\
173,179 & 178936222537081 \\
\hline
\end{tabular}

Here the (Euler) product is taken over all the primes $q$, and $f(q)$ is given below:

$$
\begin{aligned}
& f(3)= \begin{cases}3 / 2 & \text { when } a^{2} \equiv b^{2}(\bmod 9), \\
1 & \text { otherwise }\end{cases} \\
& f(q)=1 \quad \text { when } q \mid a b \\
& \text { if } q \equiv-1(\bmod 3) \text { and } q+a b, \text { then } f(q)=q^{2} /\left(q^{2}-1\right) \text {; } \\
& \text { if } q \equiv 1(\bmod 3) \text { and } q+a b, \text { then } \\
& f(q)= \begin{cases}q^{2} /(q-1)^{2} & \text { when }(D / q)_{3}=1, \\
q^{2} /\left(q^{2}+q+1\right) & \text { otherwise. }\end{cases}
\end{aligned}
$$

If we use the symbol $\Pi_{j}^{Q}$ to denote the product over all primes less than or equal to $Q$ and $\equiv j(\bmod 3)$, and if we denote by $T(Q, D)$ the infinite product

$$
\prod_{\substack{q>Q \\ q \equiv 1(\bmod 3)}} f(q)
$$

taken over all the primes exceeding $Q$ and $\equiv 1(\bmod 3)$, then, since the infinite product

$$
\prod_{q \equiv-1(\bmod 3)} f(q)
$$

taken over all the primes $\equiv-1(\bmod 3)$ converges, we have

$$
\Phi(1)=\left(f(3) \prod_{-1}^{Q} f(q) \prod_{1}^{Q} f(q)\right) T(Q, D)(1+o(1)) \text {. }
$$

Now

$$
\prod_{1}^{Q} f(q) \leqslant \prod_{1}^{Q} q^{2} /(q-1)^{2}
$$


hence

$$
\prod_{-1}^{Q} f(q) \prod_{1}^{Q} f(q) \leqslant(2 / 3) \prod^{Q} q /(q-1) \prod_{\prod}^{Q} q /(q-\chi(q)),
$$

where each of the products on the right-hand side is evaluated over all the primes $\leqslant Q$ and $\chi(q)=(-3 / q)$. By Mertens' theorem

$$
\prod^{Q} q /(q-1)=e^{\gamma} \log Q(1+o(1))
$$

Also, since

$$
L(1, \chi)=\prod_{q} q /(q-\chi(q))=\frac{\pi}{3 \sqrt{3}}
$$

(the product taken over all the primes $q$ ), we get

$$
\Phi(1) \leqslant \frac{2 \pi e^{\gamma} f(3)}{9 \sqrt{3}}(\log Q) T(Q, D)(1+o(1)) .
$$

If $\mathscr{E}$ is the extension $\mathscr{K}(\omega)$ of $\mathscr{K}$, where $\omega^{2}+\omega+1=0$, then the discriminant $d$ of $\mathscr{E}$ is $3 \Delta^{2}$ (see Barrucand and Cohn [1]). If we put

$$
U(D)=T\left((\log d)^{2}, D\right)
$$

then $U(D)<1+o(1)$ under the extended Riemann Hypothesis for $\zeta_{\mathscr{E}}$ (see, for example, Williams, Dueck and Schmid [9, pp. 282-283]). Combining this result and (3.4) with $Q=(\log d)^{2}$, we get

$$
\Phi(1)<\frac{4 \pi e^{\gamma} f(3)}{9 \sqrt{3}} \log \log \left(3 \Delta^{2}\right)(1+o(1)) .
$$

It follows from (3.3) that

$$
h R<\frac{2 e^{\gamma} f(3) \sqrt{|\Delta| / 3}}{9} \log \log \left(3 \Delta^{2}\right)(1+o(1))
$$

When, for example, $D$ is square-free, then

$$
h R< \begin{cases}(1 / 3) e^{\gamma} D \log \log 3^{3} D^{4}(1+o(1)) & \text { when } D \equiv \pm 1(\bmod 9) \\ (2 / 3) e^{\gamma} D \log \log 3^{7} D^{4}(1+o(1)) & \text { otherwise }\end{cases}
$$

4. Further Numerical Results. From (3.3) we see that in order to maximize $R$ we must minimize $h$ and get $\Phi(1)$ as large as possible. Of the possibilities for $D$ square-free, $D \neq \equiv \pm 1(\bmod 9)$ and $3+h($ see Honda [2]) we elected to examine prime values of $D \equiv 2$ or $5(\bmod 9)$. If $r_{i}$ is the $i$ th prime of the form $1+3 t$, then the prime $D$ values which should give large $\Phi(1)$ values are those for which $\left(D / r_{i}\right)_{3}=1$ $(i=1,2,3, \ldots, n)$ for as large a value of $n$ as possible. We now encounter a difficulty, however. The determination of $P$ is very expensive for rather modest values of $D$ (say $\approx 200000$ ); thus, we decided to look at the values of $R$ instead. By 
using the methods described in [9] we can calculate $R$ much more rapidly than $P$; but, it still becomes very expensive to find $R$ when $D>2 \times 10^{9}$. (It should, of course, be borne in mind that the discriminants for such values of $D$ are very large, exceeding $10^{20}$.)

UMSU was programmed to find the first 50 values of $D$ for each $n$ until a value of $n$ was reached for which the least of these 50 numbers exceeded $2^{31}-1$, the word size of the machine used to compute $R$-an AMDAHL 470-V8. We then computed $R$ for each $D$ and $C(D)=R /\left(D \log \log \left(3^{7} D^{4}\right)\right)$. In the cubic case it takes very little time to find the $D$ values and a much larger amount of time to find the $R$ values, the reverse of the situation in the quadratic case.

Our results are summarized in the following tables. In Tables 7 and 8 we give only those values of $D$ for which $C(D)$ exceeds the $C$ values for any of the other numbers that we found which were less than $D$. In Tables 9 and 10 we give values of $D$ for which the corresponding regulator exceeds any of those previously found. Since $2 e^{\gamma} / 3 \approx 1.18738$, we have nothing here that comes near to violating the Riemann Hypothesis for $\zeta_{\mathscr{E}}$. Also the growth of $C(D)$ is slow and getting slower as $D$ increases.

TABLE 7. $D \equiv 2(\bmod 9)$

\begin{tabular}{|r|r|r|}
\hline \multicolumn{1}{|c|}{$\mathrm{D}$} & \multicolumn{1}{|c|}{$\mathrm{R}$} & $\mathrm{C}(\mathrm{D})$ \\
\hline 29 & 40.27082 & .454983 \\
1721 & 3669.37913 & .588309 \\
39521 & 92172.43814 & .596085 \\
92009 & 218706.73901 & .597544 \\
343433 & 895028.71553 & .640002 \\
6616667 & 18089884.90792 & .642420 \\
7202369 & 19994005.36092 & .651564 \\
202306187 & 586455162.98256 & .653911 \\
562788101 & 1689849729.97072 & .670149 \\
\hline
\end{tabular}


TABLE $8 . D \equiv 5(\bmod 9)$

\begin{tabular}{|r|r|r|}
\hline \multicolumn{1}{|c|}{$\mathrm{D}$} & $\mathrm{R}$ & \multicolumn{1}{c|}{$\mathrm{C}(\mathrm{D})$} \\
\hline 41 & 56.28937 & .440672 \\
239 & 431.94224 & .533495 \\
1301 & 2549.94344 & .545373 \\
4523 & 9440.96250 & .560767 \\
19391 & 42811.86808 & .572868 \\
67829 & 154494.32105 & .575923 \\
72617 & 168197.50896 & .584893 \\
143879 & 361610.34278 & .626614 \\
1145327 & 3021373.73848 & .635515 \\
8596463 & 23331608.01905 & .635544 \\
8666393 & 23925356.23751 & .646390 \\
48487811 & 139358465.15040 & .658771 \\
55570523 & 163251776.10755 & .672292 \\
60435383 & 179011355.42037 & .677194 \\
\hline
\end{tabular}

TABLE $9 . D \equiv 2(\bmod 9)$

\begin{tabular}{|c|c|c|}
\hline $\mathrm{D}$ & $\mathrm{R}$ & $\mathrm{C}(\mathrm{D})$ \\
\hline 689816063 & 1888303399.286361 & .609701902921 \\
780923333 & 2040735586.012364 & .581327045940 \\
807748787 & 2264449384.076498 & .623423186470 \\
911130401 & 2663628567.917647 & .649341195701 \\
947294867 & 2666732555.238140 & .625039976249 \\
1039506833 & 2941248070.747570 & .627656937643 \\
1090062947 & 3194601736.597826 & .649803055418 \\
1250773679 & 3481668991.375506 & .616374865552 \\
1345747619 & 3810698517.456939 & .626570574715 \\
1411121837 & 3967734472.270628 & .621882987760 \\
1627729013 & 4492140541.726865 & .609547346456 \\
1695130949 & 5107010533.454052 & .665168464608 \\
2044171163 & 5464205375.038442 & .589124377005 \\
& & \\
\hline
\end{tabular}


TABLE $10 . D \equiv 5(\bmod 9)$

\begin{tabular}{|c|c|c|}
\hline $\mathrm{D}$ & $\mathrm{R}$ & $\mathrm{C}(\mathrm{D})$ \\
\hline 78446831 & 195588785.889993 & .568325353629 \\
85474661 & 229039192.818766 & .610210176803 \\
140795537 & 399133674.604591 & .641989245305 \\
172132241 & 499066717.859134 & .655156175458 \\
226496759 & 590664701.273444 & .587554486086 \\
230154107 & 639837059.815727 & .626247127877 \\
246667721 & 681500286.460078 & .621912563185 \\
258947807 & 705470723.780494 & .612941643650 \\
262192559 & 708275870.201634 & .607683348911 \\
267667889 & 739791831.127160 & .621604001102 \\
313154087 & 892931349.895030 & .640243198576 \\
613655951 & 1737626269.841794 & .631422697999 \\
641290649 & 1782982379.770936 & .619710166881 \\
671319221 & 1958397914.780726 & .649933701273 \\
736002077 & 2030844203.759158 & .614180052846 \\
784288049 & 2197173781.242724 & .623178495983 \\
789581183 & 2210040440.336767 & .622584176723 \\
792812201 & 2226806639.150783 & .624725493228 \\
860248787 & 2595846960.356864 & .670626975448 \\
914070821 & 2652552259.996093 & .644540413185 \\
948371243 & 2660317585.609716 & .622821760536 \\
957302429 & 2743025183.193182 & .636135024907 \\
1400879507 & 4155081949.704781 & .656054383521 \\
161773509 & 4322176122.273822 & .590142344485 \\
1632061859 & 4549031363.516906 & .615612610599 \\
1827261311 & 4810329644.671832 & .580807712394 \\
1831479161 & 5515724098.441698 & .664430018640 \\
2108312123 & 5713478707.454342 & .59708541322 \\
2124689657 & 6127255313.478815 & .635344945017 \\
& & \\
\hline & &
\end{tabular}

Department of Computer Science

University of Waterloo

Waterloo, Ontario, Canada N2L 3G1

Department of Computer Science

University of Manitoba

Winnipeg, Manitoba, Canada R3T 2N2

1. P. BARRUCAND \& H. COHN, "Remarks on principal factors in a relative cubic field," J. Number Theory, v. 3, 1971, pp. 226-239.

2. T. Honda, "Pure cubic fields whose class numbers are multiples of three," J. Number Theory, v. 3, 1971, pp. 7-12.

3. D. H. LeHMer, "A history of the sieve process," in A History of Computing in the Twentieth Century, Academic Press, New York, 1980, pp. 445-456.

4. D. H. Lehmer, E. Lehmer \& D. ShanKs, "Integer sequences having prescribed quadratic character," Math. Comp., v. 24, 1970, pp. 433-451.

5. C. D. Patterson \& H. C. Williams, "A report on the University of Manitoba Sieve Unit," Congr. Numer., v. 37, 1983, pp. 85-98.

6. G. F. VoronoI, On a Generalization of the Algorithm of Continued Fractions, Doctoral Dissertation, Warsaw, 1896. (Russian)

7. H. C. Williams, "A numerical investigation into the length of the period of the continued fraction expansion of $\sqrt{D}$," Math. Comp., v. 36, 1981, pp. 593-601.

8. H. C. Williams, Continued Fractions and Number Theoretic Computations, Proc. Number Theory Conf., Edmonton, 1983; Rocky Mountain J. Math. (To appear.)

9. H. C. Williams, G. W. DuecK \& B. K. SChmid, "A rapid method of evaluating the regulator and class number of a pure cubic field," Math. Comp., v. 41, 1983, pp. 235-286. 